\title{
COMPARISON OF INDIVIDUAL AND GROUP-BASED LOAD-VELOCITY PROFILING AS A MEANS TO DICTATE TRAINING LOAD OVER A SIX-WEEK STRENGTH AND POWER INTERVENTION
}

Comparison of load-velocity profiling methods to dictate training load

H. F. Dorrell, J.M Moore, and T. I. Gee

School of Sport and Exercise Science, University of Lincoln, Lincoln, UK

PURPOSE: To explore the effects of two velocity-based loading methods over a sixweek strength and power intervention in resistance trained males.

METHODS: Nineteen resistance trained males (mean \pm SD; age: $23.6 \pm 3.7$ years; stature: $182.7 \pm 5.1 \mathrm{~cm}$; body mass: $92.2 \pm 8.7 \mathrm{~kg}$ ) were recruited. Following familiarisation, participants completed two custom back squat one repetition maximum protocols (1-RM; $150.7 \pm 23.7 \mathrm{~kg}$ ), with mean concentric velocity (MCV) monitored enabling load-velocity profiles to be created. Additionally, participants completed countermovement, static squat, and standing broad jump protocols (CMJ, SSJ, and SBJ, respectively). Participants were randomly assigned to individual (ILVP; $n=9$ ) or group-based load-velocity profiling (GLVP; $n=10$ ) groups, both featuring two training sessions per week for six weeks. For the ILVP group, load and repetitions were dictated based on individual data collected during the initial load-velocity profiles. In comparison, for the GLVP intervention, load and repetitions were dictated using a generalised group-based load-velocity profile, consisting of all participant data. For both groups, relative training loads and number of sets and repetitions were equated. Following the intervention, participants retested all baseline variables. Independent sample $t$-tests were completed to examine the pre-training inter-group, and post-training intra-group differences. A two-way mixed ANOVA, using one inter-factor (ILVP vs. GLVP) and one intra-factor (pre- vs. post-training), was conducted to examine the pre to post between group differences. Cohen's $d$ effect sizes (ES) were also calculated.

RESULTS: No significant differences were present between groups at baseline. Training resulted in significant increases in 1-RM back squat for both groups (ILVP: 
9.7\%; GLVP: 7.2\%), with no group by time interaction $\left(F_{(1,17)}=3.97 p=0.06\right)$. Significant increases in CMJ, SSJ, and SBJ performance were recorded for the ILVP group (6.6\%, 4.6\%, and 6.7\%, respectively), and CMJ and SSJ only for the GLVP group (both 4.3\%). Small to moderate ES were noted for both interventions across all variables (ILVP vs. GLVP; back squat: 0.66 vs. 0.43 ; CMJ: 0.32 vs. 0.21 ; SSJ: 0.25 vs. 0.21 ; SBJ: 0.32 vs. 0.19 ).

CONCLUSION: The data presented demonstrates the potential impact of utilising a velocity-based loading approach on measures of maximal strength and power. Specifically, the results suggest that use of individualised velocity-based loading may offer a greater magnitude of change for some athletes when compared to a groupbased approach.

PRACTICAL APPLICATION: Sufficient evidence is provided supporting the use of velocity-based loading interventions within a resistance trained population. While no interaction was witnessed between groups for the back squat or jump assessments, the ILVP group did amass greater percentage increases and larger effect sizes suggesting that utilising individual differences may potentiate greater physical adaptations. Such methods appear to provide the strength and conditioning professional with greater control over prescribing load, limiting the chances of unnecessary fatigue, while providing measurable performance increases. 